\title{
Commentary: Targeting LDH enzymes with a stiripentol analog to treat epilepsy
}

\author{
Chang-Hoon Cho* \\ School of Biosystem and Biomedical Science, College of Health Science, Korea University, Seoul, South Korea
}

Keywords: epilepsy, lactate, astrocyte, lactate dehydrogenase, ketogenic diet, oxamate, mTOR, circadian

\section{A commentary on}

Targeting LDH enzymes with a stiripentol analog to treat epilepsy

by Sada, N., Lee, S., Katsu, T., Otsuki, T., and Inoue, T. (2015). Science 347, 1362-1367. doi: 10.1126/science.aaa1299

Meeting energy demands in neurons is critical for proper functions of nervous systems. In addition to glucose, lactate is used as a major energy source in the brain, and a significant amount of lactate is produced through aerobic glycolysis in astrocytes (Schurr et al., 1988; Gladden, 2004; Ivanov et al., 2011; Dienel, 2012). It is reversibly converted to and from pyruvate by lactate dehydrogenase $(\mathrm{LDH})$, and it is transported from astrocytes to neurons via the astrocyte-neuron lactate shuttle (Pellerin and Magistretti, 2012). Lactate is released from astrocytes through monocarboxylate transporters (MCT1 and MCT4), and has been also reported to be released through ion channels yet to be identified (Korn et al., 2005; Sotelo-Hitschfeld et al., 2015). Released lactate is taken into neurons through MCT2 and converted to pyruvate (Bergersen, 2007). Lactate also induces expression of genes (e.g., arc and $c$-fos) involved in synaptic plasticity (Suzuki et al., 2011; Yang et al., 2014).

OPEN ACCESS

Edited by:

Francesco Moccia,

University of Pavia, Italy

Reviewed by:

Oliver Kann,

University of Heidelberg, Germany

Alberto Spalice,

Sapienza University of Rome, Italy

*Correspondence:

Chang-Hoon Cho,

chois007@korea.ac.kr

Received: 14 April 2015 Accepted: 25 June 2015 Published: 07 July 2015

Citation:

Cho C-H (2015) Commentary:

Targeting $L D H$ enzymes with a stiripentol analog to treat epilepsy.

Front. Cell. Neurosci. 9:264. doi: 10.3389/fncel.2015.00264
In epilepsy where abnormal network activities of hyperexcitable neurons are uncontrollably synchronized, abundant energy for these activities has to be supplemented (Bertram et al., 1998). Expectedly, although more studies need to be done, high rates of glucose metabolism and elevated activity of LDH have been shown in people with epilepsy (PWE) and in animal models of epilepsy (Dufour et al., 2003). Increased level of lactate has been reported in some epilepsy cases (Hill et al., 1999). Other glycolytic enzymes are identified as markers for intractable temporal lobe epilepsies (e.g., neuron-specific enolases) and their defects (e.g., malic enzyme 2 and pyruvate dehydrogenase) are also shown either causative or susceptible to certain types of epilepsy (Steinhoff et al., 1999; Greenberg et al., 2005; Prasad et al., 2011).

Recently, Tsuyoshi Inoue's group in Japan reported that the anti-epileptic effect of the ketogenic diet (KD) bypass glycolysis (especially LDH), but occur elaborately through $\mathrm{K}_{\mathrm{ATP}}$ channelsmediated mechanisms (Ma et al., 2007; Sada et al., 2015). They have shown in electrophysiological recordings that switching glucose to ketone bodies [ $\beta$-hydroxybutyrate $(\beta-H B)$ or acetoacetate] in artificial cerebrospinal fluid hyperpolarized resting membrane potentials in excitatory neurons, and reduced the firing rate of action potentials in acute brain slice preparations. Replacing $B-$ $\mathrm{HB}$ either with glucose or lactate returned the initial level of resting membrane potentials and the firing rate. Electrophysiological data recorded when oxamate, an LDH inhibitor, was included in the recording pipette showed similar effects to the B-HB's data. This suggests that bypassing glycolysis in astrocytes, to supply energy for neurons, reduces the neuronal excitability. Paired recordings of astrocytes and neurons demonstrated that astrocytes are the site of LDH inhibition. Next, in kainate and pilocarpine models of epilepsy in mice, direct injection in the hippocampus or 
intraperitoneal injection of oxamate reduced the number of paroxysmal discharges (epileptiform activity), although the effect was short-lived (losing its drastic effect in an hour). Oxamate suppressed pilocarpine-induced acute behavioral seizures (status epilepticus) and prolonged its latency. It also suppressed paroxysmal discharges in a chronic epilepsy model. The antisense oligodeoxynucelotide targeting LDHA, one of two major genes composed of $\mathrm{LDH}$, was injected into the hippocampus of kainate model, suppressing spontaneous spikes. This confirms that $\mathrm{LDH}$ is a critical target. Next, they screened clinically available anti-epileptic drugs for inhibiting activities of purified LDH, and found that Stiripentol partially inhibits LDH. Isosafrole, a Stiripentol derivative that has a greater $\mathrm{LDH}$ inhibitory effect than Stiripentol does, suppressed spontaneous spikes dramatically in kainate model (Sada et al., 2015). This is a significant step forward elucidating the metabolic mechanism of epilepsy. Although LDH is not the first metabolic enzyme shown to be important in epilepsy, its inhibition was the first to suppress the epileptiform discharges in vivo (Novarino et al., 2012; Papetti et al., 2013).

In epilepsy, hypoxia-inducing factor $1 \alpha(\mathrm{HIF} 1 \alpha)$ has been induced in reactive astrocytes (Vangeison et al., 2008; Li et al., 2014). Several glycolytic enzymes, including $\mathrm{LDH}$, have been shown to be upregulated by HIF- $1 \alpha$, although HIF- $1 \alpha$-mediated $\mathrm{LDH}$ induction needs to be verified in PWE and animal models of epilepsy (Marín-Hernández et al., 2009). Interestingly, oxamate inhibits not only LDH but also mTOR pathway, which is the major signaling pathway in both genetic and acquired epilepsies (Cho, 2011; Zhao et al., 2015). In addition, activation of mTOR pathway increases the expression of LDH by activating STAT3, a transcription factor and downstream target of mTOR pathway, which has been shown to be activated in epilepsy

\section{References}

Bergersen, L. H. (2007). Is lactate food for neurons? Comparison of monocarboxylate transporter subtypes in brain and muscle. Neuroscience 145, 11-19. doi: 10.1016/j.neuroscience.2006.11.062

Bertram, E. H., Zhang, D. X., Mangan, P., Fountain, N., and Rempe, D. (1998). Functional anatomy of limbic epilepsy: a proposal for central synchronization of a diffusely hyperexcitable network. Epilepsy Res. 32, 194-205. doi: 10.1016/S0920-1211(98)00051-5

Cho, C. H. (2011). Frontier of epilepsy research - mTOR signaling pathway. Exp. Mol. Med. 43, 231-274. doi: 10.3858/emm.2011.43.5.032

Cho, C. H. (2012). Molecular mechanism of circadian rhythmicity of seizures in temporal lobe epilepsy. Front. Cell. Neurosci. 6:55. doi: 10.3389/fncel.2012.00055

Dienel, G. A. (2012). Brain lactate metabolism: the discoveries and the controversies. J. Cereb. Blood Flow Metab. 32, 1107-1138. doi: $10.1038 /$ jcbfm.2011.175

Dufour, F., Koning, E., and Nehlig, A. (2003). Basal levels of metabolic activity are elevated in Genetic Absence Epilepsy Rats from Strasbourg (GAERS): measurement of regional activity of cytochrome oxidase and lactate dehydrogenase by histochemistry. Exp. Neurol. 182, 346-352. doi: 10.1016/S0014-4886(03)00052-9

Fisher, J. L. (2009). The anti-convulsant stiripentol acts directly on the GABA(A) receptor as a positive allosteric modulator. Neuropharmacology 56, 190-197. doi: 10.1016/j.neuropharm.2008.06.004
(Lund et al., 2008; Zha et al., 2011). Furthermore, rapamycin, an mTOR inhibitor, reduces lactate level by decreasing the activity and expression of $\mathrm{LDH}$ and/or inhibiting hexokinase II, an upstream enzyme of glycolysis (Venkatesh et al., 2012; Lee et al., 2013). Therefore, there are interactions between the mTOR pathway and lactate/LDH, which need to be explored further. Finally, metabolic enzymes in glycolysis are regulated in a circadian manner (Zhang et al., 2009). Particularly, LDH activity and its mRNA expression follow the circadian pattern in suprachiasmatic nucleus (Isobe et al., 2011). It is entertaining to imagine that if the level of lactate in the brain is higher at night than during the day, nocturnal types of epilepsy might be better explained (Isobe et al., 2011; Cho, 2012).

There are many follow-up questions that remain to be addressed. First, what would be the side-effect of prolonged inhibition of LDH for controlling epileptic seizures? Will cells in other organs (e.g., heart and muscle) function properly with LDH inhibitors? If not, how we can make this work only on (reactive) astrocytes in the epileptic foci? Second, will isosafrole have enhancing effects of GABAergic synaptic transmission like stiripentol (Quilichini et al., 2006; Fisher, 2009; Grosenbaugh and Mott, 2013)? Third, although lactate in the hippocampus has been shown to be decreased by KD without affecting the level and the activity of $\mathrm{LDH}$ in this study, it remains to be seen that the level of lactate is lowered by KD in PWE and animal models of epilepsy.

\section{Acknowledgments}

The author is pleased to acknowledge Dr. M. McCartney's comments on the manuscript.
Gladden, L. B. (2004). Lactate metabolism: a new paradigm for the third millennium. J. Physiol. 558(Pt 1), 5-30. doi: 10.1113/jphysiol.2003.058701

Greenberg, D. A., Cayanis, E., Strug, L., Marathe, S., Durner, M., Pal, D. K., et al. (2005). Malic enzyme 2 may underlie susceptibility to adolescentonset idiopathic generalized epilepsy. Am. J. Hum. Genet. 76, 139-146. doi: $10.1086 / 426735$

Grosenbaugh, D. K., and Mott, D. D. (2013). Stiripentol is anticonvulsant by potentiating GABAergic transmission in a model of benzodiazepinerefractory status epilepticus. Neuropharmacology 67, 136-143. doi: 10.1016/j.neuropharm.2012.11.002

Hill, R. A., Chiappa, K. H., Huang-Hellinger, F., and Jenkins, B. G. (1999). Hemodynamic and metabolic aspects of photosensitive epilepsy revealed by functional magnetic resonance imaging and magnetic resonance spectroscopy. Epilepsia 40, 912-920. doi: 10.1111/j.1528-1157.1999.tb00798.x

Isobe, Y., Hida, H., and Nishino, H. (2011). Circadian rhythm of metabolic oscillation in suprachiasmatic nucleus depends on the mitochondrial oxidation state, reflected by cytochrome C oxidase and lactate dehydrogenase. J. Neurosci. Res. 89, 929-935. doi: 10.1002/jnr.22609

Ivanov, A., Mukhtarov, M., Bregestovski, P., and Zilberter, Y. (2011). Lactate effectively covers energy demands during neuronal network activity in neonatal hippocampal slices. Front. Neuroenergetics 3:2. doi: 10.3389/fnene.2011.00002

Korn, T., Magnus, T., and Jung, S. (2005). Interaction with antigen-specific T cells regulates expression of the lactate transporter MCT1 in primary rat astrocytes: specific link between immunity and homeostasis. Glia 49, 73-83. doi: 10.1002/glia.20101 
Lee, S. C., Marzec, M., Liu, X., Wehrli, S., Kantekure, K., Ragunath, P. N., et al. (2013). Decreased lactate concentration and glycolytic enzyme expression reflect inhibition of mTOR signal transduction pathway in B-cell lymphoma. NMR Biomed. 26, 106-114. doi: 10.1002/nbm.2825

Li, J., Jiang, G., Chen, Y., Chen, L., Li, Z., Wang, Z., et al. (2014). Altered expression of hypoxia-Inducible factor- $1 \alpha$ participates in the epileptogenesis in animal models. Synapse 68, 402-409. doi: 10.1002/syn.21752

Lund, I. V., Hu, Y., Raol, Y. H., Benham, R. S., Faris, R., Russek, S. J., et al. (2008). BDNF selectively regulates GABAA receptor transcription by activation of the JAK/STAT pathway. Sci. Signal. 1:ra9. doi: 10.1126/scisignal.1162396

Marín-Hernández, A., Gallardo-Pérez, J. C., Ralph, S. J., Rodríguez-Enríquez, S., and Moreno-Sánchez, R. (2009). HIF-1alpha modulates energy metabolism in cancer cells by inducing over-expression of specific glycolytic isoforms. Mini Rev. Med. Chem. 9, 1084-1101.

Ma, W., Berg, J., and Yellen, G. (2007). Ketogenic diet metabolites reduce firing in central neurons by opening K(ATP) channels. J. Neurosci. 27, 3618-3625. doi: 10.1523/JNEUROSCI.0132-07.2007

Novarino, G., El-Fishawy, P., Kayserili, H., Meguid, N. A., Scott, E. M., Schroth, J., et al. (2012). Mutations in BCKD-kinase lead to a potentially treatable form of autism with epilepsy. Science 338, 394-397. doi: 10.1126/science.1224631

Papetti, L., Parisi, P., Leuzzi, V., Nardecchia, F., Nicita, F., Ursitti, F., et al. (2013). Metabolic epilepsy: an update. Brain Dev. 35, 827-841. doi: 10.1016/j.braindev.2012.11.010

Pellerin, L., and Magistretti, P. J. (2012). Sweet sixteen for ANLS. J. Cereb. Blood Flow Metab. 32, 1152-1166. doi: 10.1038/jcbfm.2011.149

Prasad, C., Rupar, T., and Prasad, A. N. (2011). Pyruvate dehydrogenase deficiency and epilepsy. Brain Dev. 33, 856-865. doi: 10.1016/j.braindev.2011.08.003

Quilichini, P. P., Chiron, C., Ben-Ari, Y., and Gozlan, H. (2006). Stiripentol, a putative antiepileptic drug, enhances the duration of opening of GABA-A receptor channels. Epilepsia 47, 704-716. doi: 10.1111/j.15281167.2006.00497.x

Sada, N., Lee, S., Katsu, T., Otsuki, T., and Inoue, T. (2015). Epilepsy treatment. Targeting LDH enzymes with a stiripentol analog to treat epilepsy. Science 347, 1362-1367. doi: 10.1126/science.aaa1299

Schurr, A., West, C. A., and Rigor, B. M. (1988). Lactate-supported synaptic function in the rat hippocampal slice preparation. Science 240, 1326-1328. doi: 10.1126/science. 3375817

Sotelo-Hitschfeld, T., Niemeyer, M. I., Mächler, P., Ruminot, I., Lerchundi, R., Wyss, M. T., et al. (2015). Channel-mediated lactate release by k+-stimulated astrocytes. J. Neurosci. 35, 4168-4178. doi: 10.1523/JNEUROSCI.5036-14.2015
Steinhoff, B. J., Tumani, H., Otto, M., Mursch, K., Wiltfang, J., Herrendorf, G., et al. (1999). Cisternal S100 protein and neuron-specific enolase are elevated and site-specific markers in intractable temporal lobe epilepsy. Epilepsy Res. 36, 75-82. doi: 10.1016/S0920-1211(99)00026-1

Suzuki, A., Stern, S. A., Bozdagi, O., Huntley, G. W., Walker, R. H., Magistretti, P. J., et al. (2011). Astrocyte-neuron lactate transport is required for long-term memory formation. Cell 144, 810-823. doi: 10.1016/j.cell.2011.02.018

Vangeison, G., Carr, D., Federoff, H. J., and Rempe, D. A. (2008). The good, the bad, and the cell type-specific roles of hypoxia inducible factor- 1 alpha in neurons and astrocytes. J. Neurosci. 28, 1988-1993. doi: 10.1523/JNEUROSCI.532307.2008

Venkatesh, H. S., Chaumeil, M. M., Ward, C. S., Haas-Kogan, D. A., James, C. D., and Ronen, S. M. (2012). Reduced phosphocholine and hyperpolarized lactate provide magnetic resonance biomarkers of PI3K/Akt/mTOR inhibition in glioblastoma. Neuro Oncol. 14, 315-325. doi: 10.1093/neuonc/nor209

Yang, J., Ruchti, E., Petit, J. M., Jourdain, P., Grenningloh, G., Allaman, I., et al. (2014). Lactate promotes plasticity gene expression by potentiating NMDA signaling in neurons. Proc. Natl. Acad. Sci. U.S.A. 111, 12228-12233. doi: 10.1073/pnas.1322912111

Zha, X., Wang, F., Wang, Y., He, S., Jing, Y., Wu, X., et al. (2011). Lactate dehydrogenase B is critical for hyperactive mTOR-mediated tumorigenesis. Cancer Res. 71, 13-18. doi: 10.1158/0008-5472.CAN-10-1668

Zhang, E. E., Liu, A. C., Hirota, T., Miraglia, L. J., Welch, G., Pongsawakul, P. Y., et al. (2009). A genome-wide RNAi screen for modifiers of the circadian clock in human cells. Cell 139, 199-210. doi: 10.1016/j.cell.2009.08.031

Zhao, Z., Han, F., Yang, S., Wu, J., and Zhan, W. (2015). Oxamate-mediated inhibition of lactate dehydrogenase induces protective autophagy in gastric cancer cells: involvement of the Akt-mTOR signaling pathway. Cancer Lett. 358, 17-26. doi: 10.1016/j.canlet.2014.11.046

Conflict of Interest Statement: The author declares that the research was conducted in the absence of any commercial or financial relationships that could be construed as a potential conflict of interest.

Copyright (C) 2015 Cho. This is an open-access article distributed under the terms of the Creative Commons Attribution License (CC BY). The use, distribution or reproduction in other forums is permitted, provided the original author (s) or licensor are credited and that the original publication in this journal is cited, in accordance with accepted academic practice. No use, distribution or reproduction is permitted which does not comply with these terms. 